\title{
Van der Waals Spin Valves
}

\author{
C. Cardoso, D. Soriano, N. A. García-Martínez, and J. Fernández-Rossier* \\ QuantaLab, International Iberian Nanotechnology Laboratory (INL), Avenida Mestre José Veiga, 4715-330 Braga, Portugal
}

(Received 9 April 2018; published 7 August 2018)

\begin{abstract}
We propose spin valves where a 2D nonmagnetic conductor is intercalated between two ferromagnetic insulating layers. In this setup, the relative orientation of the magnetizations of the insulating layers can have a strong impact on the in-plane conductivity of the $2 \mathrm{D}$ conductor. We first show this for a graphene bilayer, described with a tight-binding model, placed between two ferromagnetic insulators. In the antiparallel configuration, a band gap opens at the Dirac point, whereas in the parallel configuration, the graphene bilayer remains conducting. We then compute the electronic structure of graphene bilayer placed between two monolayers of the ferromagnetic insulator $\mathrm{CrI}_{3}$, using density functional theory. Consistent with the model, we find that a gap opens at the Dirac point only in the antiparallel configuration.
\end{abstract}

DOI: 10.1103/PhysRevLett.121.067701

The controlled fabrication of layered structures combining ferromagnetic conductors and nonmagnetic materials, thin enough as to preserve spin polarization, made possible the discovery of fundamental spin-dependent transport phenomena, such as giant magnetoresistance [1,2] and tunnel magnetoresistance $[3,4]$. These developments led to the concept of a spin valve, a structure whose conductivity is modulated by the relative orientation of two ferromagnetic electrodes [5] and, altogether, set the foundations of spintronics.

The study of the so-called van der Waals heterostructures $[6,7]$, metamaterials obtained by vertical stacking of 2D crystals, is a very fertile area of research. Using relatively simple fabrication methods, they allow the study of structures with tailored electronic properties that combine a variety of 2D materials, including insulators (h-BN), semiconductors $\left(\mathrm{MoS}_{2}\right)$, conductors (graphene), and superconductors $\left(\mathrm{NbSe}_{2}\right)$. The recent discovery of $2 \mathrm{D}$ crystals with magnetic order [8-11] adds both ferromagnetic and antiferromagnetic insulators to this list. For instance, van der Waals devices incorporating atomically thin layers of the ferromagnetic insulator $\mathrm{CrI}_{3}$ have been reported [12-17]. These findings pave the way to van der Waals spintronics with new types of spin-dependent transport phenomena.

Here we propose a new class of spin valve that takes advantage of the spin proximity effect, i.e., the spin polarization of the surface electrons of a nonmagnetic material adjacent to a ferromagnet. The proposed system, depicted in Fig. 1, consists of a 2D conducting crystal sandwiched between two insulating ferromagnetic layers. If the magnetizations of the two proximity layers are antiparallel (AF), the spin proximity effects have opposite sign at both sides of the 2D crystal. In contrast, for the parallel state (FM), the top and bottom proximity effects add up. As we show below, this difference has a strong influence in the in-plane conductance of the nonmagnetic conductor, and in some instances leads to a conductor to insulator transition in the $2 \mathrm{D}$ crystal. This strong influence of spin proximity effect in a 2D crystal contrasts with the case of 3D materials, for which proximity effects are constrained only to their surface.

Our proposal is different from lateral graphene spin valves $[18,19]$, where large areas of graphene are not in contact with the ferromagnetic electrodes, and is also different from spin-filter tunnel junctions, where the magnetic insulators act as barrier materials for vertical transport $[12-14,20]$. The proposed spin valve resembles the early current in plane structures where giant magnetoresistance was discovered [1,2], with the obvious difference that the magnetic layers are insulating in the van der Waals spin valves.

We first illustrate the concept of van der Waals spin valves considering the case when the central conductor is a graphene bilayer. We use the standard tight-binding model

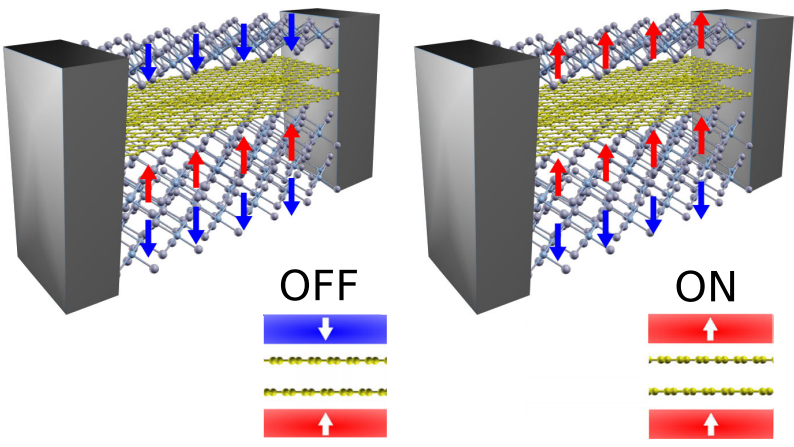

FIG. 1. Van der Waals spin valve, where a conducting 2D crystal is sandwiched between two insulating ferromagnets. Lateral contacts can drive in-plane current. The magnetization of the bottom ferromagnetic layer is pinned, whereas the top layer can switch, resulting in two configurations, (a) antiparallel (AF) and (b) parallel (FM), with very different in-plane conductance. 
for the graphene bilayer [21]. Proximity effects have been reported both for graphene monolayers and bilayers [2225]. A spin proximity effect is considered [26] by including a spin-dependent potential $\Delta$ whose sign can be different in the top and bottom graphene layers. Previous density functional theory (DFT) calculations for monolayer graphene deposited on different ferromagnetic insulators, such as EuO [27,28], EuS [28], and YIG [28], justify this model. We assume that the magnetization of both top and bottom layers lie on the same axis. The only relevant angle in this problem is the one formed by the magnetizations of top and bottom layers. We also assume that the bottom magnetization does not change, resulting in fixed spin-dependent potential $\sigma \Delta$, where $\sigma$ is the spin projection along the magnetization axis. The top layer spin-dependent potential is given by $\eta \sigma \Delta$, where $\eta= \pm 1$ describes the magnetization orientation of the top magnetic layer, relative to the bottom layer. Thus, $\eta=+1$ describes the parallel (FM) orientation and $\eta=-1$ the antiparallel (AF) case.

Represented in the basis $A_{1}, B_{1}, A_{2}, B_{2}$, where $A$ and $B$ correspond to the two triangular sublattices, and the subindices 1 and 2 label the top and bottom layers, respectively, the Bloch Hamiltonian for spin $\sigma$ states reads

$$
\mathcal{H}_{\sigma}(\vec{k})=\left(\begin{array}{cccc}
\eta \sigma \frac{\Delta}{2} & f(\vec{k}) & 0 & 0 \\
f^{*}(\vec{k}) & \eta \sigma \frac{\Delta}{2} & \gamma & 0 \\
0 & \gamma & \sigma \frac{\Delta}{2} & f(\vec{k}) \\
0 & 0 & f(\vec{k})^{*} & \sigma \frac{\Delta}{2}
\end{array}\right)
$$

where $f(\vec{k})=t\left(1+e^{i \vec{k} \cdot \vec{a}_{1}}+e^{i \vec{k} \cdot \vec{a}_{2}}\right)$ and $\gamma$ describe the intralayer and interlayer hopping matrix elements, respectively. The resulting spin resolved energy bands, in the neighbourhood of the Dirac point, are shown in Fig. 2 for the two states of the spin valve, $\eta= \pm 1$. For the FM alignment $(\eta=+1)$, the graphene bilayer presents spinsplit bands, and remains in a conducting state, i.e., with a finite density of states at the Fermi energy. In contrast, for the AF case $(\eta=-1)$, a band gap opens up at the Dirac point. Thus, depending on the relative alignment of the top and bottom insulating ferromagnets, the graphene bilayer spin valve can be either a conductor, for the FM alignment, or a gapped system with depleted conductance, when the Fermi energy is set at the Dirac point. Within this model, both the band gap in the AF alignment and the spin splitting in the FM alignment are given by $\Delta$.

We now address the origin of the gap opening in graphene bilayer AF alignment of the van der Waals spin valve. For each spin channel, the Hamiltonian (1) in the AF alignment is identical to the model of the graphene bilayer with an off-plane electric field, that is known to open up a gap in the band structure [29,30]. Interestingly, in the spin valve, the sign of the effective electric field is opposite for opposite spins, $E_{\text {eff }} \propto \sigma \Delta$. The spin projection of the AF bands over top and bottom layers, shown in Fig. 2, clearly

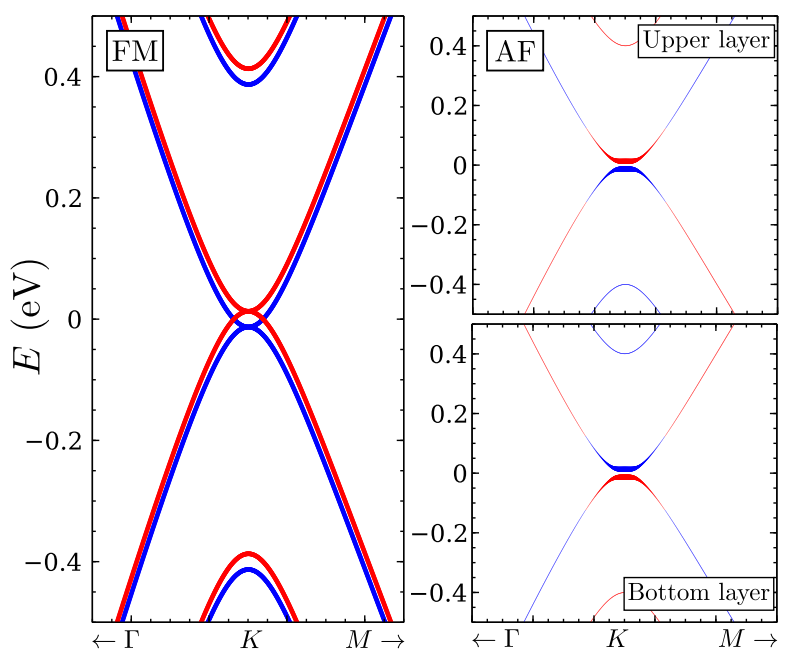

FIG. 2. Bands structure computed with model Hamiltonian for the FM (left) and AF (right) configurations, Red and blue denote different spin states. For the AF state, the bands have been projected in the upper and lower layer of the graphene bilayer, with the size of the dots proportional to the layer polarization, revealing that the top of the valence band and bottom of the conduction band are spin polarized in each of the layers.

shows the presence of a spin dipole [31]: for a given spin, there is a charge imbalance driven by the exchange with the magnetic layers that is compensated exactly by the opposite spin.

In a graphene bilayer, the gap opened by an electric field is known to have a valley-dependent Chern number $\mathcal{C}=K \operatorname{sgn}(E)$, where $K= \pm 1$ labels the valleys [32,33]. In the case of the spin valve in the AF state, this leads to Chern numbers that are both spin and valley dependent:

$$
\mathcal{C}=K \sigma= \pm 1 \text {. }
$$

Equation (2) permits us to anticipate [32,33] the emergence of one-dimensional spin-dependent chiral in-gap states in domain walls separating two antiferromagnetic domains with opposite magnetizations [see Fig. 3(b)]. In order to verify this, we compute the momentum resolved density of states of a domain wall along the zigzag direction as ilustrated in Fig. 3(b). The domain wall is assumed to be abrupt, preserving spin collinearity. The calculation is done for a system with translational invariance along the wall direction, and embedded between two semi-infinite gapped graphene bilayer planes, using a Green's function technique [34]. Both domains are insulating, but at each valley the Chern number is opposite for a given spin direction. Thus, a domain wall along the zigzag direction, that preserves the valley, features two chiral 1D in-gap states per valley and per spin [see Fig. 3(a)]. It is interesting to note that, for a given valley, the states are spin chiral and therefore backscattering requires either spin mixing, or intervalley scattering. A noncollinear domain wall might result in spin mixing. 


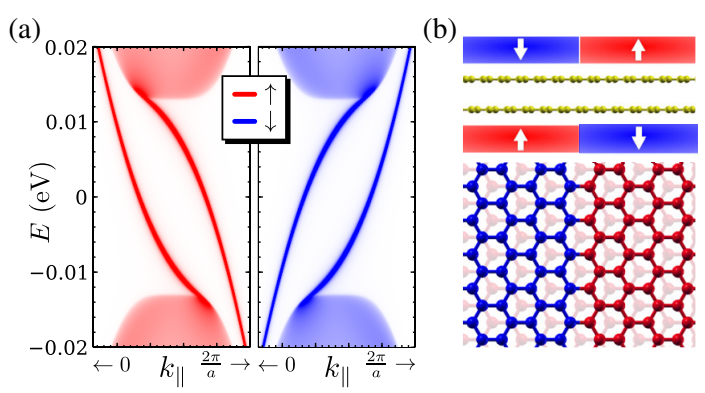

FIG. 3. (a) Momentum resolved density of states revealing chiral in-gap states, for a given valley, computed for the domain wall between two insulating antiferromagnetic domains with opposite spin orientation (b). The velocity of the bands changes sign in the opposite valley.

We now consider a possible physical realization of the Van der Waals spin valve, based on a graphene bilayer, that is feasible within the experimental state of the art. For that matter, we choose $\mathrm{CrI}_{3}$ monolayers as the insulating ferromagnet. It was recently shown that $\mathrm{CrI}_{3}$ monolayers remain ferromagnetic up to $45 \mathrm{~K}$ [11]. In addition $\mathrm{CrI}_{3}$ preserves its magnetic properties even when deposited on graphite [11] or encapsulated between graphite electrodes [12-14]. Moreover, the spin proximity effect between $\mathrm{CrI}_{3}$ and two-dimensional $\mathrm{WSe}_{2}$ has been demonstrated experimentally [35].

The DFT calculations were performed using QUANTUM ESPRESSO [36] with PBE exchange-correlation potential [37] and projector augmented wave (PAW) pseudopotentials [38,39] and including van der Waals interactions within the semiempirical method of Grimme (DFT-D2) [40]. Spin orbit interactions, known to be important to determine the magnetic anisotropy of $\mathrm{CrI}_{3}$ [41], are not included in the calculation as they are not expected to induce qualitative change in the spin proximity effect discussed here.

We now discuss our results both for monolayer $\mathrm{CrI} 3$ and monolayer graphene as well as graphene bilayer in between two monolayers of $\mathrm{CrI}_{3}$. The former permits us to rationalize the results of the bilayer. For the bilayer, we use a unit cell with 4 layers, $\mathrm{CrI}_{3} /$ graphene bilayer $/ \mathrm{CrI}_{3}$. We use a lattice parameter along $z$ that ensures the absence of interaction between the replicas of the system. In all cases the unit cell contains a $3 \times 3$ supercell with 18 carbon atoms per graphene layer, and 2 chromium and 6 iodine atoms per $\mathrm{CrI}_{3}$ layer. Thus, for the sandwiched graphene bilayer, the unit cell has a total of 52 atoms. The calculations show that the spin of $\mathrm{Cr}$ atoms is $S=3 / 2$, which are hosted by the $t_{2 g}$ bands.

We first discuss the results for the monolayer graphene on top of $\mathrm{CrI}_{3}$, shown in Fig. 4(a). Our results are in line with previous DFT calculations for this system [42]. With the exception of some anticrossings, the energy bands are an overlay of those of the decoupled monolayers, as expected in a van der Waals structure. Occupied bands, way below the Fermi energy $E_{F}$, are made of iodine $p$ states and the spin majority $t_{2 g}$ states of Cr. Empty bands, high above $E_{F}$, are made of spin minority $t_{2 g}$ states of $\mathrm{Cr}$. In the $2 \mathrm{eV}$ window around the Fermi energy, the bands are those of the graphene Dirac cones and the spin majority $e_{g}$ states. These four bands (coming from $2 \mathrm{Cr}$ atoms in the unit cell) are narrow, lie almost completely above the Fermi energy, and hybridize with the graphene Dirac cones in the majority spin channel close to the Dirac point. In contrast, the minority spin Dirac cone remains intact [see illustration in Fig. 4(b)]. Therefore, for one spin channel the Dirac electrons barely notice the presence of the $\mathrm{CrI}_{3}$; for the other spin channel, there is a strong hybridization with a narrow band.

The bands structure of the $\mathrm{CrI}_{3} /$ graphene bilayer/ $\mathrm{CrI}_{3}$ are shown in Figs. 4(c) and 4(d), both for the FM (c) and AF (d) configurations. Both of them show the graphene bilayer bands and the $e_{g}$ bands. For the FM case, the shape of the graphene bilayer bands is preserved in one spin channel (the minority spin), but a strong hybridization opens up a gap in the majority spin channel, slightly above the Fermi energy, which lies below the Dirac point. For the AF configuration, both spin channels of the graphene bilayer become hybridized with the narrow $e_{g}$ bands of $\mathrm{CrI}_{3}$. So, from that point of view alone, we expect that the
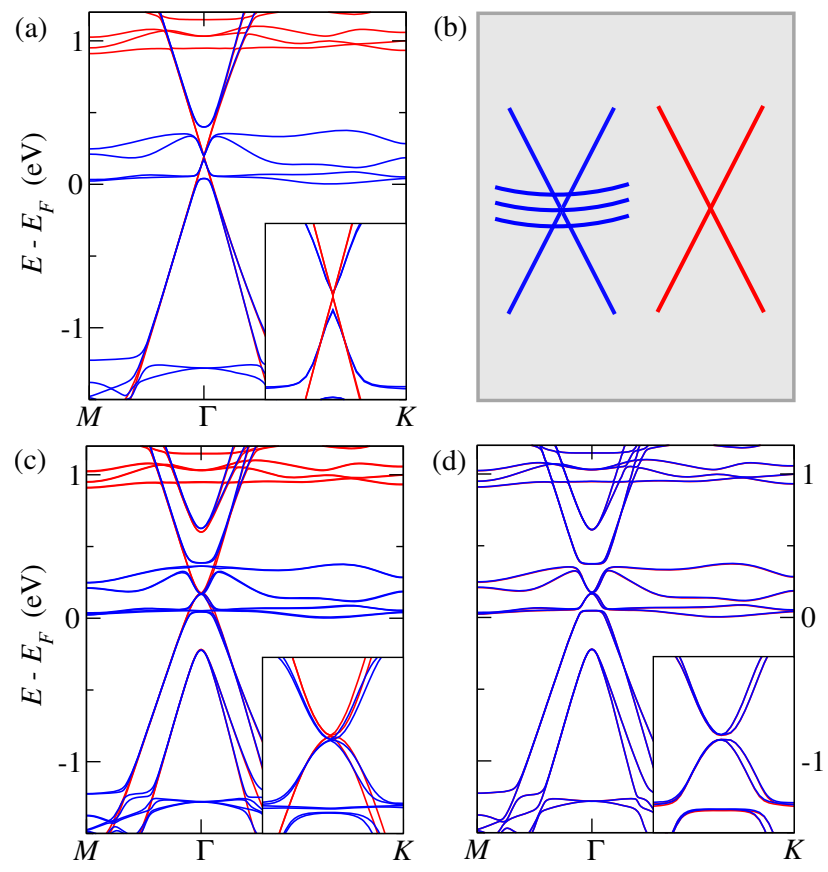

FIG. 4. (a) Band structure, for the graphene monolayer on top of a $\mathrm{CrI}_{3}$ monolayer. (b) Scheme of energy bands for graphene monolayer on $\mathrm{CrI}_{3}$. (c),(d) Band structure for grahene bilayer placed between ferromagnetic monolayers of $\mathrm{CrI}_{3}$ with $\mathrm{FM}$ (c) and $\mathrm{AF}(\mathrm{d})$ relative alignment. The insets show a closeup around the Dirac point, showing a spin splitting in the FM alignment and a gap for the AF case, both taking the same value of $7 \mathrm{meV}$. 
in-plane conductance is much larger in the FM alignment than the $\mathrm{AF}$ one.

We now turn our attention to the states around the Dirac energy, where the conduction and valence parabolic bands of freestanding graphene bilayer meet. For the AF configuration, a band gap of $7 \mathrm{meV}$ splits the electron and hole parabolic bands of graphene bilayer [see inset of Fig. 4(d)]. For the FM configuration, there is a spin splitting of the bands near the Dirac point, whose magnitude is, interestingly, the same as the AF gap. The sign of the effective Crgraphene exchange obtained in the DFT calculation is ferromagnetic: the sign of the spin splitting of the graphene bilayer bands at the Dirac point is the same than the $\mathrm{CrI}_{3}$ spin splitting. The origin and sign of this coupling is discussed in the Supplemental Material [43]. In this regard, the DFT results for the bilayer graphene placed between two $\mathrm{CrI}_{3}$ layers are in line with the toy model described above. However, there is electron transfer from graphene to $\mathrm{CrI}_{3}$, so that the Fermi energy does not lie at the Dirac point. It must be noticed that, even if there is charge transfer, we expect a very small in-plane conductance in the $\mathrm{CrI}_{3}$, on account of the very small dispersion of the occupied states.

Thus, our DFT calculations strongly suggest that a graphene bilayer encapsulated between two layers of $\mathrm{CrI}_{3}$ will present a strong spin-valve effect, with the FM alignment having a larger in-plane conductance, on account of the fact that bands in one spin channel are decoupled from the nondispersive $\mathrm{CrI}_{3}$ bands, in contrast with the AF, where both spin channels are hybridized. Our calculation assumes a full saturation of the magnetization, valid for temperatures much smaller than the Curie temperature. In addition, the thermal occupation of the bands across the band gap would also diminish the spin valve effect. Interestingly, the same mechanism should also apply for the graphene monolayer. In addition, for the graphene bilayer, application of both a top and a bottom gate voltage could set the Fermi energy at the Dirac point, resulting in a conductor to insulator transition driven by the alignment of the magnetizations.

The control of the relative orientation of the magnetization of the layers could be done by application of a magnetic field, provided that two conditions are met. First, the interlayer coupling should be smaller than the Zeeman coupling. Interlayer coupling of $\mathrm{CrI}_{3}$ bilayers, without graphene in the middle, meets this demand. The presence of the graphene bilayer should significantly reduce the interlayer coupling. Accordingly, our DFT calculations yield $J<10 \mu \mathrm{eV}$ per unit cell. Second, the switching field of top and bottom layers should be different. A very natural way to achieve that is to pin the magnetization of the bottom layer. This could be done, for instance, using a bilayer of $\mathrm{CrI}_{3}$ for which antiferromagnetic interlayer coupling has been reported [11,12,15-17]
The concept of van der Waals spin valve goes beyond the case of the graphene bilayer. For instance, the tunable spin proximity effect can drive a metal insulator transition in a graphene monolayer in the quantum Hall regime. At half filling, high quality graphene quantum Hall bars are often insulating. Application of a strong in-plane magnetic field can induce a quantum Hall ferromagnet that has a finite edge conductance [34,44]. In a spin valve, such a transition could be promoted by a spin proximity effect, rather than Zeeman interaction. Another possibility is van der Waals spin valves with a superconducting middle layer, such as $\mathrm{NbSe}_{2}$. In the FM state, spin proximity effect can kill superconductivity, which would only survive in the AF state. Such a transition has been observed in superconducting thin films sandwiched between bulk ferromagnetic insulators [45]. The concept of van der Waals's spin valve can be extended to the control of optical properties. For instance, spin proximity might control whether dark or bright excitons of the middle layer are the ground state of the $2 \mathrm{D}$ semiconductor.

In conclusion, we have proposed a new type of spin valve where a nonmagnetic 2D crystal is sandwiched between two ferromagnetic insulators and its in-plane conductance is controlled by a spin proximity effect. We hope that our work will motivate the experimental exploration of van der Waals current in plane spin valves, including the other materials in the nonmagnetic layers, such as superconductors, as well as other magnetic layers, such as bulk ferrromagnets, for which the spin valve effect proposed here should also work.

We acknowledge financial support from the European Regional Development Fund Project No. NORTE-0150145-FEDER-000019, the Marie Curie Nano TRAIN for Growth Cofund program at the International Iberian Nanotechnology Laboratory and Fundação para a Ciência e a Tecnologia for the P2020-PTDC/FIS-NAN/3668/2014 project. We acknowledge J. L. Lado for fruitful discussions and technical assistance in the calculations. We acknowledge Efrén Navarro-Morata for fruitful discussions.

On leave from Departamento de Física Aplicada, Universidad de Alicante, Spain.

[1] M. N. Baibich, J. M. Broto, A. Fert, F. Nguyen Van Dau, F. Petroff, P. Etienne, G. Creuzet, A. Friederich, and J. Chazelas, Phys. Rev. Lett. 61, 2472 (1988).

[2] G. Binasch, P. Grünberg, F. Saurenbach, and W. Zinn, Phys. Rev. B 39, 4828 (1989).

[3] M. Julliere, Phys. Lett. A 54, 225 (1975).

[4] J. S. Moodera, L. R. Kinder, T. M. Wong, and R. Meservey, Phys. Rev. Lett. 74, 3273 (1995).

[5] B. Dieny, V. S. Speriosu, S. Metin, S. S. Parkin, B. A. Gurney, P. Baumgart, and D. R. Wilhoit, J. Appl. Phys. 69, 4774 (1991).

[6] A. K. Geim and I. V. Grigorieva, Nature (London) 499, 419 (2013). 
[7] K. Novoselov, A. Mishchenko, A. Carvalho, and A. C. Neto, Science 353, aac9439 (2016).

[8] X. Wang, K. Du, Y. Y. F. Liu, P. Hu, J. Zhang, Q. Zhang, M. H. S. Owen, X. Lu, C. K. Gan, P. Sengupta et al., 2D Mater. 3, 031009 (2016).

[9] J.-U. Lee, S. Lee, J. H. Ryoo, S. Kang, T. Y. Kim, P. Kim, C.-H. Park, J.-G. Park, and H. Cheong, Nano Lett. 16, 7433 (2016).

[10] C. Gong, L. Li, Z. Li, H. Ji, A. Stern, Y. Xia, T. Cao, W. Bao, C. Wang, Y. Wang et al., Nature (London) 546, 265 (2017).

[11] B. Huang, G. Clark, E. Navarro-Moratalla, D. R. Klein, R. Cheng, K. L. Seyler, D. Zhong, E. Schmidgall, M. A. McGuire, D. H. Cobden et al., Nature (London) 546, 270 (2017).

[12] D. R. Klein, D. MacNeill, J. L. Lado, D. Soriano, E. Navarro-Moratalla, K. Watanabe, T. Taniguchi, S. Manni, P. Canfield, J. Fernández-Rossier et al., Science 360, 1218 (2018).

[13] T. Song, X. Cai, M. W.-Y. Tu, X. Zhang, B. Huang, N. P. Wilson, K. L. Seyler, L. Zhu, T. Taniguchi, K. Watanabe et al., Science 360, 1214 (2018).

[14] Z. Wang, I. Gutiérrez-Lezama, N. Ubrig, M. Kroner, T. Taniguchi, K. Watanabe, A. Imamoğlu, E. Giannini, and A. F. Morpurgo, Nat. Commun. 9, 2516 (2018).

[15] S. Jiang, J. Shan, and K. F. Mak, Nat. Mater. 17, 406 (2018).

[16] B. Huang, G. Clark, D. R. Klein, D. MacNeill, E. Navarro-Moratalla, K. L. Seyler, N. Wilson, M. A. McGuire, D. H. Cobden, D. Xiao et al., Nat. Nanotechnol. 13, 544 (2018).

[17] S. Jiang, L. Li, Z. Wang, K. F. Mak, and J. Shan, Nat. Nanotechnol. 13, 549 (2018).

[18] N. Tombros, C. Jozsa, M. Popinciuc, H. T. Jonkman, and B. J. Van Wees, Nature (London) 448, 571 (2007).

[19] W. Han, R. K. Kawakami, M. Gmitra, and J. Fabian, Nat. Nanotechnol. 9, 794 (2014).

[20] G.-X. Miao, M. Müller, and J. S. Moodera, Phys. Rev. Lett. 102, 076601 (2009).

[21] F. Guinea, A. H. Castro Neto, and N. M. R. Peres, Phys. Rev. B 73, 245426 (2006).

[22] Z. Wang, C. Tang, R. Sachs, Y. Barlas, and J. Shi, Phys. Rev. Lett. 114, 016603 (2015).

[23] P. Wei, S. Lee, F. Lemaitre, L. Pinel, D. Cutaia, W. Cha, F. Katmis, Y. Zhu, D. Heiman, J. Hone, J. S. Moodera, and C. Chen, Nat. Mater. 15, 711 (2016).

[24] J. C. Leutenantsmeyer, A. A. Kaverzin, M. Wojtaszek, and B. J. van Wees, 2D Mater. 4, 014001 (2017).

[25] S. Singh, J. Katoch, T. Zhu, K.-Y. Meng, T. Liu, J. T. Brangham, F. Yang, M. E. Flatté, and R. K. Kawakami, Phys. Rev. Lett. 118, 187201 (2017).
[26] V. T. Phong, N. R. Walet, and F. Guinea, 2D Mater. 5, 014004 (2017).

[27] H. X. Yang, A. Hallal, D. Terrade, X. Waintal, S. Roche, and M. Chshiev, Phys. Rev. Lett. 110, 046603 (2013).

[28] A. Hallal, F. Ibrahim, H. Yang, S. Roche, and M. Chshiev, 2D Mater. 4, 025074 (2017).

[29] T. Ohta, A. Bostwick, T. Seyller, K. Horn, and E. Rotenberg, Science 313, 951 (2006).

[30] E. McCann, Phys. Rev. B 74, 161403 (2006).

[31] J. Fernández-Rossier, Phys. Rev. B 77, 075430 (2008).

[32] I. Martin, Y. M. Blanter, and A. F. Morpurgo, Phys. Rev. Lett. 100, 036804 (2008).

[33] P. San-Jose, E. Prada, E. McCann, and H. Schomerus, Phys. Rev. Lett. 102, 247204 (2009).

[34] J. L. Lado, N. García-Martínez, and J. Fernández-Rossier, Synth. Met. 210, 56 (2015).

[35] D. Zhong, K. L. Seyler, X. Linpeng, R. Cheng, N. Sivadas, B. Huang, E. Schmidgall, T. Taniguchi, K. Watanabe, M. A. McGuire et al., Sci. Adv. 3, e1603113 (2017).

[36] P. Giannozzi, S. Baroni, N. Bonini, M. Calandra, R. Car, C. Cavazzoni, D. Ceresoli, G. L. Chiarotti, M. Cococcioni, I. Dabo et al., J. Phys.: Condens. Matter 21, 395502 (2009).

[37] J. P. Perdew, M. Ernzerhof, and K. Burke, J. Chem. Phys. 105, 9982 (1996).

[38] P. E. Blöchl, Phys. Rev. B 50, 17953 (1994).

[39] E. Kucukbenli, M. Monni, B. Adetunji, X. Ge, G. Adebayo, N. Marzari, S. De Gironcoli, and A. D. Corso, arXiv: 1404.3015.

[40] S. Grimme, J. Comput. Chem. 27, 1787 (2006).

[41] J. L. Lado and J. Fernández-Rossier, 2D Mater. 4, 035002 (2017).

[42] J. Zhang, B. Zhao, T. Zhou, Y. Xue, C. Ma, and Z. Yang, Phys. Rev. B 97, 085401 (2018).

[43] See Supplemental Material at http://link.aps.org/ supplemental/10.1103/PhysRevLett.121.067701 for details on the origin of exchange interaction between bilayer Graphene and $\mathrm{CrI}_{3}$, as drawn from a 4-bands toy model which takes into account the hybridization of bilayer graphene bands with spin-polarized $\mathrm{CrI}_{3}$ flat bands. With this model we can easily anticipate the results obtained using density functional theory calculations for parallel or anti-parallel alignment of $\mathrm{CrI}_{3}$ magnetic layers.

[44] A. Young, J. Sanchez-Yamagishi, B. Hunt, S. Choi, K. Watanabe, T. Taniguchi, R. Ashoori, and P. Jarillo-Herrero, Nature (London) 505, 528 (2014).

[45] B. Li, N. Roschewsky, B. A. Assaf, M. Eich, M. EpsteinMartin, D. Heiman, M. Münzenberg, and J. S. Moodera, Phys. Rev. Lett. 110, 097001 (2013). 